\title{
Triple Primary Origin Tumor: A Case Report
}

\author{
Yong-Su Jung, Se-Hoon Kim, Sung-Kon Ha, Sang-Dae Kim, Dong-Jun Lim \\ Department of Neurosurgery, Ansan Hospital, Korea University School of Medicine, Ansan, Republic of Korea
}

Generally, among the extradural spinal tumors, metastatic spinal tumor is much more common than primary spinal tumors. Thus, in the case of a spinal tumor patient with cancer history (such as lung cancer, breast cancer, etc.), we used to infer that the spinal lesion is the metastasis from, primary malignancy. We introduce an experience of a case of triple primary origin tumor in a 57-year-old man. When the spinal lesion was found on the abdominal computed tomography scan, he already had a history of colon cancer and liver cancer. Initially, it was thought that the lesion would probably be a metastatic tumor from the liver or colon cancers, and the operation was performed accordingly. In the pathologic final report, however, the mass was proven to plasmacytoma - the third primary lesion. The patient underwent chemotherapy after surgery. Globally, the triple primary origin tumor has been reported very rarely. With this report, we wish to emphasize the necessity of pathologic confirmation and adequate treatment even in a patient with known malignancies.

Key Words: Triple primary origin tumor $\cdot$ Spinal metastasis $\cdot$ Primary spinal tumor $\cdot$ Pathologic diagnosis

\section{INTRODUCTION}

Spinal tumors are known to be relatively rare among neoplastic diseases. But as the expected length of life extends every year and due to the rapid development in diagnostic tools, the incidence of spinal tumors is increasing markedly. As a result, many studies on spinal tumors are under progress. Spinal tumors often do not accompany symptoms, but as it progresses, it may accompany muscle weakness, pain, or even serious disability such as quadriplegia. Therefore, like other tumors, early discovery and treatment may have an absolute impact on the prognosis of the patient ${ }^{2,6}$. Generally, for spinal tumors, metastatic spinal tumor is known to be much more common than primary spinal tumor. Therefore, once a spinal tumor is identified on imaging studies such as CT scan or MRI, full metastasis work up for finding a probable primary location of the tumor is essential. Especially more in case of patients with a previous cancer history (lung cancer, breast cancer, or else). It is easier to regard the spinal tumor as metastatic lesion from the existing tumors of other organs.

\footnotetext{
- Received: March 12, 2013 - Revised: May 23, 2013

- Accepted: May 31, 2013

Corresponding Author: Se-Hoon Kim, MD

Department of Neurosurgery, Korea University Ansan Hospital, 516,

Gojan-dong, Danwon-gu, Ansan 425-707, Republic of Korea

Tel: +82-31-412-5053, Fax: +82-31-412-5054

E-mail:sean1050@gmail.com
}

\section{CASE REPORT}

An abdominal CT scan was taken of a 57-year-old male patient who had sacral and right buttock and thigh pain, and revealed with a tumor-like lesion at the T12 vertebrae. Previously in 2007 , the patient had been diagnosed with colon cancer, confirmed as adenocarcinoma by open biopsy. Targeting the cancer lesion, only endoscopic mucosal resection was executed at that time and was considered as a cure. After a period of 3 years, on a follow up large intestine endoscope, a recurred lesion was identified, thus abdominal low anterior tumor resection with colostomy was performed immediately. The patient was also diagnosed with liver cancer in 2009, which was confirmed as hepatocellular carcinoma. About this hepatic lesion, the patient was treated by radio-frequency ablation twice.

The T12 level tumor mass was first discovered in the abdominal CT which was taken to confirm the hepatic lesion, and in the follow-up abdominal CT executed 6 months later, the diameter of tumor mass was increased from $1.5 \mathrm{~cm}$ to $4 \mathrm{~cm}$ (Fig. 1, 2). First, it was presumed that the lesion might be a metastatic lesion originated from the primary colon or liver cancer considering the previous cancer history of the patient. As a treatment for the vertebral lesion, total laminectomy, right hemi-vertebrectomy of T12 and tumor resection was performed, and pedicle screw fixation of T10, T11, L1, and L2 vertebral bodies were done. Observed intra-operatively, 


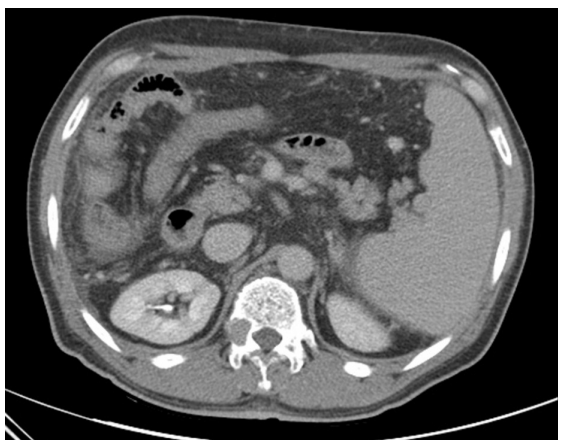

Fig. 1. T12 vertebral body lesion was first discovered by the abdomen CT conducted for liver cancer, which was approximately $1.5 \mathrm{~cm}$ in diameter. The tumor mass invaded T12 vertebral body, pedicle, and facet joint mainly in right side.
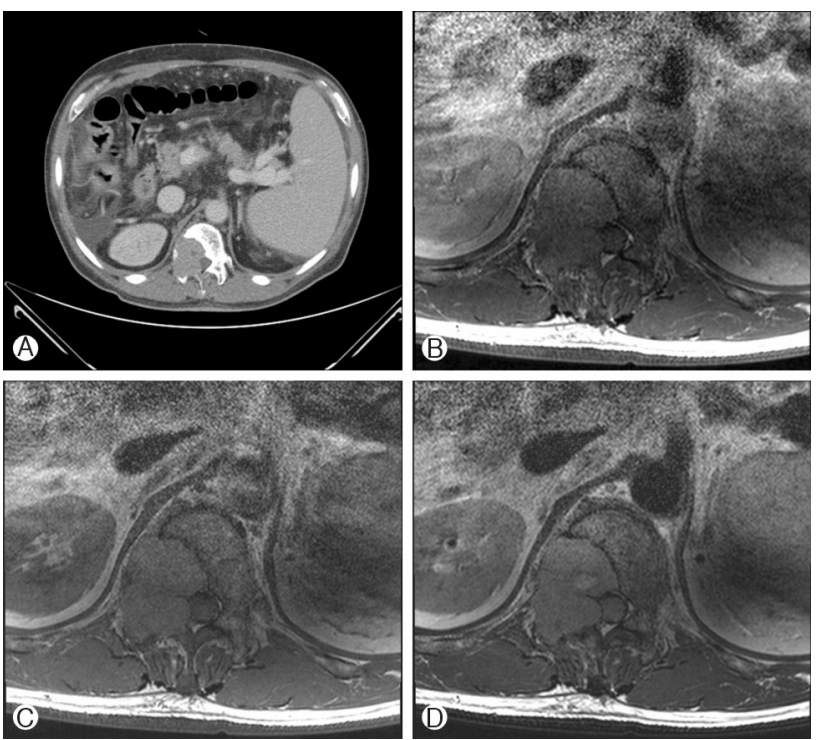

Fig. 2. In the follow-up abdomen CT and MRI which were performed 6 month later, the previously discovered T12 level tumor mass was found to have grown to $4 \mathrm{~cm}$ in diameter. The tumor demonstrated slightly increased signal intensity in T1- and T2-weighted images, and enhanced faintly.

the tumor was found to be a brownish, hypervascular, and friable mass compressing the thecal sac on right side of T11-12 level, and additional erosion of the T12 lamina was also identified.

On the final pathologic report, however, it was confirmed as plasmacytoma (Fig. 3, 4), which was completely different pathologically from previously diagnosed colon cancer or liver cancer. On additional immunostaining, the tumor cells also showed a monoclonality for lambda immunoglobulin in its light chain.

In conclusion, the patient was finally diagnosed to have triple primary-origin tumors of adenocarcinoma, hepatocellular

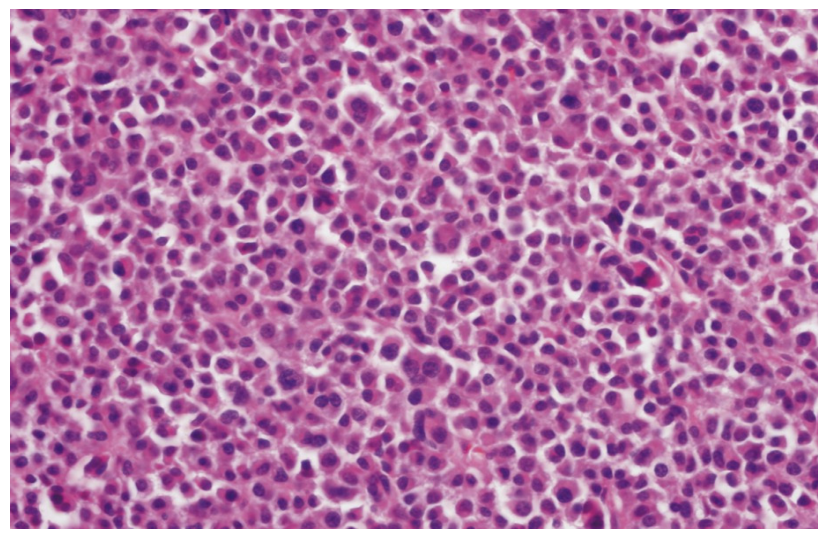

Fig. 3. Plasma cells are seen in the specimen (Hematoxylin \& Eosin stain, $\times 400$ ).

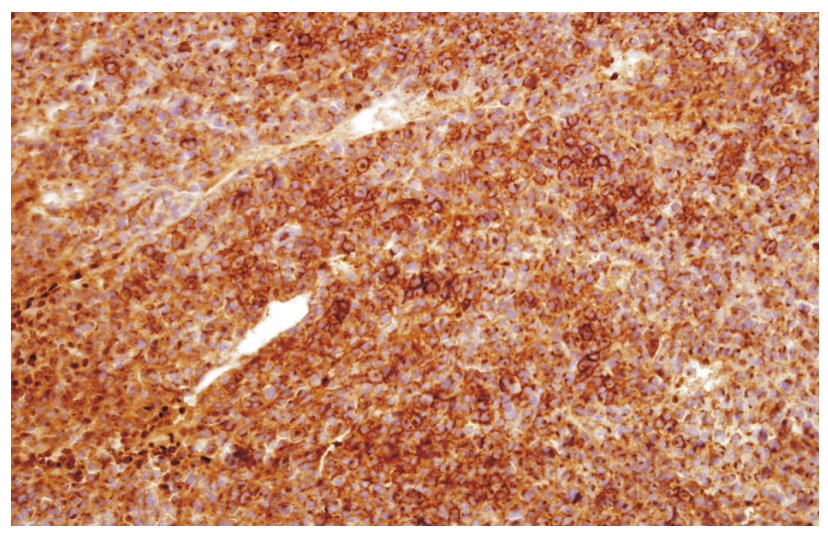

Fig. 4. CD138 stain revealed scattered plasma cells (CD138 stain, $\times 400)$.

carcinoma, and vertebral plasmacytoma. The patient underwent chemotherapy after the surgery.

\section{DISCUSSION}

Generally, spinal tumors are mostly metastatic tumors, and those that often cause metastasis to spine include lung cancer, breast cancer, and prostate cancer. On the contrary, primary spinal tumor is very rare so that among the most representative of such, multiple myeloma is found in 1-2 persons per $100,000^{1)}$, and chordoma is found in 0.5 persons per $100,000^{3)}$. Therefore, when a patient is found with a lesion suspicious of a tumor in the spine, the expectation is that it is a metastatic lesion and the metastatic examination and treatment are conducted, and in most cases the future biopsy results are consistent. Under such circumstances, when a lesion suspicious of spinal tumor is found in a patient already diagnosed with tumors in other organs, the probability and the suspicion that this lesion is a spinal metastasis from an existing tumor are bound 
to be even greater.

As in our case above, however, there clearly is the case where the spinal lesion is primary and a completely different type of tumor is diagnosed despite having two different types of tumors in different organs, and the treatment directions may differ completely depending on this.

For example, when a lesion suspicious of tumor is found in spine from the imaging study executed with the backache as the main symptom for a lung cancer patient, generally it is considered the spinal metastasis of the lung cancer and only palliative radiation therapy may be executed. If the actual spinal lesion was chordoma, however, the treatment method must be completely different - it is resistant to general radiation therapy, so the treatment direction should be surgical removal and proton-beam radiation ${ }^{4,5}$.

For this reason, it is emphasized that even if the discovered spinal lesion is suspicious of being a metastatic lesion, histological confirmation should be executed when possible.

\section{CONCLUSION}

Triple primary-origin tumor is a very rare case across the world; this report intended to cover the case report and also the necessity of histological confirmed diagnosis of spinal tumor. So far, it is difficult to make a perfect differential diag- nosis with imaging study alone without a biopsy for modern technology, and this may be resolved with development of imaging and interpretation technologies in the future.

With this report, we wish to emphasize the necessity of pathologic confirmation and adequate further treatment even in a patient with known malignancies.

\section{REFERENCES}

1. Anderson KC, Alsina M, Bensinger W, Biermann JS, ChananKhan A, Comenzo RL, et al.: Multiple myeloma. Clinical practice guidelines in oncology. J Natl Compr Canc Netw 5:118-147, 2007

2. Bilsky MH, Lis E, Raizer J, Lee H, Boland P: The diagnosis and treatment of metastatic spinal tumor. Oncologist 4:459-469, 1999

3. Ferraresi V, Nuzzo C, Zoccali C, Marandino F, Vidiri A, Salducca $\mathrm{N}$, et al.: Chordoma: clinical characteristics, management and prognosis of a case series of 25 patients. BMC Cancer 10:22, 2010

4. Imai R, Kamada T, Sugahara S, Tsuji H, Tsujii H: Carbon ion radiotherapy for sacral chordoma. Br J Radiol, 2011

5. Sciubba DM, Cheng JJ, Petteys RJ, Weber KL, Frassica DA, Gokaslan ZL: Chordoma of the sacrum and vertebral bodies. J Am Acad Orthop Surg 17:708-717, 2009

6. Xu H, Wang Y, Qiu G, Ye Q, Zhang J: Surgical treatment of metastatic spinal tumor. Chin Med Sci J 17:183-188, 2002 